\title{
Design of Pure Propagation Unscented Kalman Filter
}

\author{
Ondřej Straka, Jindřich Duník and Miroslav Šimandl \\ Department of Cybernetics and \\ European Centre of Excellence - New Technologies for the Information Society \\ Faculty of Applied Sciences, University of West Bohemia, \\ Univerzitní 8, 30614 Pilsen, Czech Republic \\ e-mails: \{straka30,dunikj,simandl\}@kky.zcu.cz,(O. Straka, J. Duník, M. Šimandl)
}

\begin{abstract}
The paper deals with state estimation of nonlinear stochastic dynamic systems. Various unscented Kalman filter (UKF) algorithms are analyzed with the focus on computation and transformation of the $\sigma$-points for the purpose of update of state estimate moments. An algorithm of the pure propagation $\mathrm{UKF}$ is developed transforming the initial $\sigma$-point set forward in time without necessity of its recomputation as is usual in classical UKF algorithms. Such direct transformation of the $\sigma$-points keeps higher order moments of the $\sigma$-point set and leads consequently to an increased accuracy of the state estimate. The proposed pure propagation unscented Kalman filter is illustrated in a numerical example.
\end{abstract}

Keywords: State estimation; Kalman filtering; Unscented Kalman filter; Higher-order moments

\section{INTRODUCTION}

State estimation of stochastic dynamic discrete-time systems is an essential tool for many traditional areas such as navigation, tracking, positioning, change detection and optimal control.

The system is characterized by a state, which is an underlying, usually immeasurable variable describing the system. The state is observed through a measurement and both exhibit uncertain behavior. Hence, the description of the system, consisting of a state dynamics and a measurement-to-state relation, is stochastic. As the state is random, the complete description of its estimate, given the measurements, is represented by a conditional probability density function (PDF). An estimate of such form is provided by the Bayesian recursive relations (BRRs).

In many situations, point estimates, such as the conditional mean of the state, are sufficient. Obtaining the point estimates by first calculating the PDF is cumbersome as usually the BRRs are intractable and their approximate solutions are largely computationally expensive (Arulampalam et al., 2002).

Another approach to obtain a point estimate of the state is represented by an optimization of a suitable criterion, such as the mean square error (MSE). Rapid development of this approach is tied with the design of the Kalman filter (KF) (Kalman, 1960) which was proposed as an optimal state estimator for linear systems minimizing the MSE criterion. Consequently, many other filtering techniques following the Kalman filtering framework have been proposed usually with the aim to relax the linearity assumptions of the KF. With a few exceptions the techniques provide an approximate solution only.

Some techniques proceed from an approximation of the nonlinear model (e.g., the extended Kalman filter, difference fil-

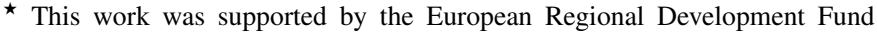
(ERDF), project NTIS - New Technologies for Information Society, European Centre of Excellence, CZ.1.05/1.1.00/02.0090 and by the Czech Science Foundation, project no. GACR P103-13-07058J.
}

ters, stochastic regression filters (Šimandl and Duník, 2009; Nørgaard et al., 2000; Ito and Xiong, 2000; Sarmavuori and Sarkka, 2012)), others from approximate computation of the predictive and filtering state estimates, i.e., the conditional means and covariance matrices appearing in the Kalman filtering framework (quadrature filter (Jia et al., 2011), cubature filter (Arasaratnam and Haykin, 2009), unscented Kalman filter (UKF) (Julier et al., 2000; Duník et al., 2012)).

The latter group of filters approximate the state estimate by a set of $\sigma$-points. The set is usually designed to capture the first two moments of the state estimate. In spite of capability of the set of storing information also about higher order moments, this information is discarded by the filter. The cause is a step performed once or twice at each time instant, which recomputes the set to update its moment (Kolås et al., 2009). As a result, only the first two moments of the state estimate are kept.

The goal of the paper is to develop a new UKF based filter with a $\sigma$-point set keeping higher order moments across time instants. This behavior will be enabled by replacing the modifications of moments by direct modifications of the $\sigma$-points. Hence, the filter will consist mainly of the $\sigma$-point propagation.

The paper is organized as follows: system specification, state estimation and the UKF will be introduced in Section 2. A technique for a direct modification of the $\sigma$-points to expand their covariance matrix will be proposed in Section 3 and the new filter will be introduced in Section 4. In Section 5 a numerical illustration of the new filter will be given and concluding remarks are drawn in Section 6.

\section{STATE ESTIMATION AND UKF}

\subsection{System Specification and State Estimation}

Consider the discrete-time nonlinear stochastic system 


$$
\begin{aligned}
\mathbf{x}_{k+1} & =\mathbf{f}_{k}\left(\mathbf{x}_{k}\right)+\mathbf{w}_{k}, \quad k=0,1,2, \ldots, \\
\mathbf{z}_{k} & =\mathbf{h}_{k}\left(\mathbf{x}_{k}\right)+\mathbf{v}_{k}, \quad k=0,1,2, \ldots,
\end{aligned}
$$

where the variables $\mathbf{x}_{k} \in \mathbb{R}^{n_{x}}$ and $\mathbf{z}_{k} \in \mathbb{R}^{n_{z}}$ represent the state and the measurement at time instant $k$, respectively, $\mathbf{f}_{k}: \mathbb{R}^{n_{x}} \rightarrow$ $\mathbb{R}^{n_{x}}$ and $\mathbf{h}_{k}: \mathbb{R}^{n_{x}} \rightarrow \mathbb{R}^{n_{z}}$ are known nonlinear functions, and $\mathbf{w}_{k}, \mathbf{w}_{k} \in \mathbb{R}^{n_{x}}$ and $\mathbf{v}_{k}, \mathbf{v}_{k} \in \mathbb{R}^{n_{z}}$ are zero-mean state and measurement white noises with known covariance matrices $\Sigma_{k}^{w}$ and $\Sigma_{k}^{v}$, respectively. The noises are assumed to be mutually independent and independent of the initial state $\mathbf{x}_{0}$ given by its mean $\overline{\mathbf{x}}_{0}$ and covariance matrix $\mathbf{P}_{0}^{x x}$.

State estimation based on MSE optimization leads to the estimate $\hat{\mathbf{x}}_{k \mid k}$ being the conditional mean

$$
\hat{\mathbf{x}}_{k \mid k}=\mathrm{E}\left[\mathbf{x}_{k} \mid \mathbf{z}^{k}\right]
$$

which is usually analytically intractable. The symbol $\mathbf{z}^{k}$ denotes a set of all measurements up to the time $k, \mathbf{z}^{k}=\left[\mathbf{z}_{0}, \mathbf{z}_{1}, \ldots \mathbf{z}_{k}\right]$.

To cope with the intractability, the estimator structure is often constrained to be a linear function of the measurement. For linear systems, the MSE optimization with the constraint leads to the KF. For nonlinear systems a further approximation is required. Either approximation of the nonlinear functions in the system description or approximation of the state estimate description (e.g., by a set of weighted points as in the unscented transform (UT) (Julier et al., 2000; Duník et al., 2012) or using integration rules (Ito and Xiong, 2000; Jia et al., 2011; Arasaratnam and Haykin, 2009)) are convenient.

\subsection{Unscented Kalman Filter}

The structure of the UKF algorithm for the system described by (1) and (2) is summarized by Algorithm 1 (Julier et al., 2000; Śimandl and Duník, 2009). For convenient purposes, the following notation will be used: $\mathcal{X}^{a: b}=\left[\mathcal{X}^{a}, \mathcal{X}^{a+1}, \ldots, \mathcal{X}^{b}\right]$, $\mathbf{1}_{a \times b}$ and $\mathbf{0}_{a \times b}$ represent matrices of ones and zeros, respectively, of indicated dimension and $\sqrt{\mathbf{P}}$, denotes a matrix decomposition of $\mathbf{P}$ such that $\mathbf{P}=\sqrt{\mathbf{P}} \sqrt{\mathbf{P}}^{\mathrm{T}}$.

Further, suppose two sets of $\sigma$-points $X^{0: 2 n_{x}} y^{0: 2 n_{x}}$ are given together with a set of weights $\mathcal{W}^{0: 2 n_{x}}$. Then, approximate calculation of the mean based on the $\sigma$-point sets given by

$$
\hat{\mathbf{x}}=\sum_{i=0}^{2 n_{x}} \mathcal{W}^{i} X^{i}
$$

will be further denoted by expression $\hat{\mathbf{x}}=\sigma-\operatorname{MEAN}(\mathcal{W}, \mathcal{X})$. Approximate calculation of a mutual covariance based on the $\sigma$-point sets given by the relation

$$
\mathbf{P}^{x y}=\sum_{i=0}^{2 n_{x}} w^{i}\left(\mathcal{X}^{i}-\hat{\mathbf{x}}\right)\left(\mathcal{Y}^{i}-\hat{\mathbf{y}}\right)^{\mathrm{T}},
$$

where $\hat{\mathbf{x}}=\sigma-\operatorname{MEAN}(\mathcal{W}, \mathcal{X})$ and $\hat{\mathbf{y}}=\sigma-\operatorname{MEAN}(\mathcal{W}, \mathcal{Y})$, will be further denoted as $P^{x y}=\sigma-\operatorname{COV}(\mathcal{W}, \mathcal{X}, \mathcal{y})$.

\section{Algorithm 1: Unscented Kalman Filter}

Step 1: (initialization) Set the time instant $k=0$.

Step 2: (prediction)

If $k=0$, let $\hat{\mathbf{x}}_{0 \mid-1}=\mathrm{E}\left[\mathbf{x}_{0}\right]=\overline{\mathbf{x}}_{0}, \mathbf{P}_{0 \mid-1}^{x x}=\operatorname{cov}\left[\mathbf{x}_{0}\right]=\mathbf{P}_{0}^{x x}$.

If $k>0$, calculate the $\sigma$-points

$$
X_{k-1 \mid k-1}^{0: 2 n_{x}}=\hat{\mathbf{x}}_{k-1 \mid k-1} \mathbf{1}_{1 \times b}+c\left[\mathbf{0}_{n_{x} \times 1}, \sqrt{\mathbf{P}_{k-1 \mid k-1}^{x x}},-\sqrt{\mathbf{P}_{k-1 \mid k-1}^{x x}}\right]
$$

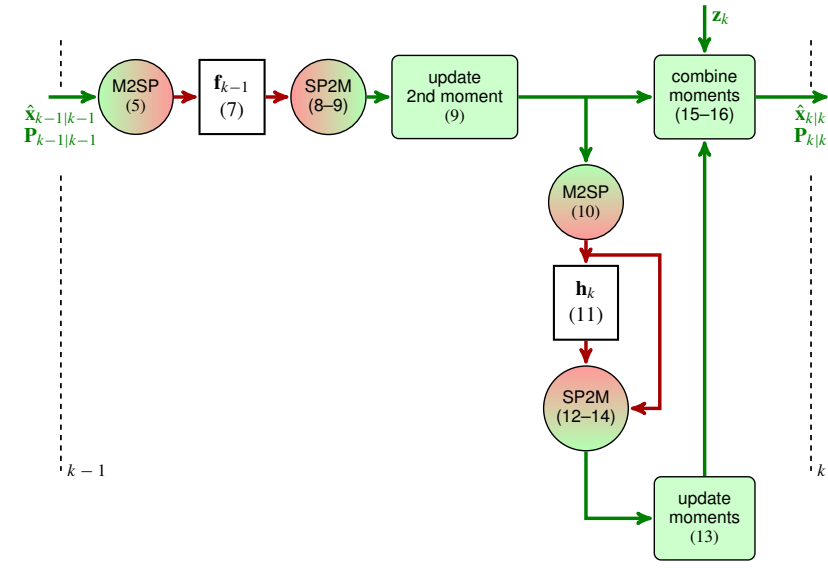

Fig. 1. Block scheme of the UKF algorithm.

with corresponding weights

$$
\mathcal{W}^{0: 2 n_{x}}=\frac{1}{n_{x}+\kappa}\left[\kappa, \frac{1}{2}, \ldots, \frac{1}{2}\right]
$$

where $b=2 n_{x}+1$ is the number of $\sigma$-points and $c=$ $\sqrt{n_{x}+\kappa}$ with $\kappa$ being the scaling factor determined by the user. Propagate each $\sigma$-point through the nonlinear function as

$$
\bar{X}_{k \mid k-1}^{i}=\mathbf{f}_{k-1}\left(X_{k-1 \mid k-1}^{i}\right), \forall i,
$$

and calculate the state predictive moments as

$$
\begin{aligned}
\hat{\mathbf{x}}_{k \mid k-1} & =\sigma-\operatorname{MEAN}\left(\mathcal{W}, \overline{\mathcal{X}}_{k \mid k-1}\right) \\
\mathbf{P}_{k \mid k-1}^{x x} & =\sigma-\operatorname{COV}\left(\mathcal{W}, \bar{\chi}_{k \mid k-1},, \bar{X}_{k \mid k-1}\right)+\Sigma_{k-1}^{w},
\end{aligned}
$$

Note that addition of $\Sigma_{k-1}^{w}$ in (9) represents the moment modification caused by appearance of $\mathbf{w}_{k}$ in (1).

Step 3: (filtering) Calculate the $\sigma$-points

$$
X_{k \mid k-1}^{0: 2 n_{x}}=\hat{\mathbf{x}}_{k \mid k-1} \mathbf{1}_{1 \times b}+c\left[\mathbf{0}_{n_{x} \times 1}, \sqrt{\mathbf{P}_{k \mid k-1}^{x x}},-\sqrt{\mathbf{P}_{k \mid k-1}^{x x}}\right] .
$$

Propagate each $\sigma$-point through the nonlinear function as

$$
Z_{k \mid k-1}^{i}=\mathbf{h}_{k}\left(X_{k \mid k-1}^{i}\right), \forall i
$$

and calculate the measurement predictive moments as

$$
\begin{aligned}
\hat{\mathbf{z}}_{k \mid k-1} & =\sigma-\operatorname{MEAN}\left(\mathfrak{W}, \mathfrak{Z}_{k \mid k-1}\right), \\
\mathbf{P}_{k \mid k-1}^{z z} & =\sigma-\operatorname{COV}\left(\mathfrak{W}, \mathfrak{Z}_{k \mid k-1}, \mathfrak{Z}_{k \mid k-1}\right)+\Sigma_{k}^{v}, \\
\mathbf{P}_{k \mid k-1}^{x z} & =\sigma-\operatorname{COV}\left(\mathfrak{W}, \boldsymbol{X}_{k \mid k-1}, \mathfrak{Z}_{k \mid k-1}\right) .
\end{aligned}
$$

Note again that (13) represents computation of the second moment and its modification by adding $\Sigma_{k}^{v}$. The filtering estimate given by the mean and covariance matrix is computed as

$$
\begin{aligned}
\hat{\mathbf{x}}_{k \mid k} & =\hat{\mathbf{x}}_{k \mid k-1}+\mathbf{K}_{k}\left(\mathbf{z}_{k}-\hat{\mathbf{z}}_{k \mid k-1}\right), \\
\mathbf{P}_{k \mid k}^{x x} & =\mathbf{P}_{k \mid k-1}^{x x}-\mathbf{K}_{k} \mathbf{P}_{k \mid k-1}^{z z} \mathbf{K}_{k}^{T},
\end{aligned}
$$

where $\mathbf{K}_{k}=\mathbf{P}_{k \mid k-1}^{x z}\left(\mathbf{P}_{k \mid k-1}^{z z}\right)^{-1}$.

Let $k=k+1$. The algorithm then continues by Step 2 .

The algorithm is illustrated in Fig. 1, where M2SP and SP2M denotes moments to a $\sigma$-point set conversion (such as in (10)) and vice-versa (such as in (12-14), respectively. It is evident that the $\sigma$-points are generated at each time instant twice, i.e., in (5) and (10). Each generation is preceded by a modification to the moments that are used to generate the new set of $\sigma$-points (i.e., (9) in the prediction step and (15) and (16) in the filtering step) to account for uncertainties of the noises.

Recomputing the $\sigma$-point set after the modification to the moments discards any higher order moment from the original 


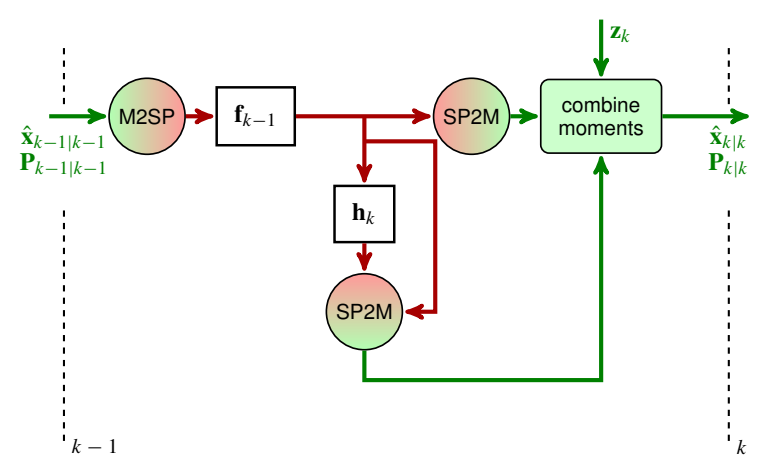

Fig. 2. Block scheme of the FAUKF algorithm.

$\sigma$-point set (van der Merwe, 2004). To prevent such disposal of the third moment information, van der Merwe (2004) proposed an algorithm of the fully augmented UKF (FAUKF).

\subsection{Fully Augmented UKF}

The FAUKF is based on augmenting the state with variables representing the state noise $\mathbf{w}_{k}$ and the measurement noise as ${ }^{A} \mathbf{x}_{k}^{\mathrm{T}} \triangleq\left[\mathbf{x}_{k}^{\mathrm{T}}, \mathbf{w}_{k}^{\mathrm{T}}, \mathbf{v}_{k+1}^{\mathrm{T}}\right]^{\mathrm{T}}$. The $\sigma$-points defined as $\left({ }^{A} X_{k \mid k}^{i}\right)^{\mathrm{T}}=$ $\left[\left({ }^{x} \mathcal{X}_{k \mid k}^{i}\right)^{\mathrm{T}},\left({ }^{w} \mathcal{X}_{k \mid k}^{i}\right)^{\mathrm{T}},\left({ }^{v} \mathcal{X}_{k \mid k}^{i}\right)^{\mathrm{T}}\right]^{\mathrm{T}}$ are generated according to the mean ${ }^{A} \hat{\mathbf{x}}_{k \mid k-1}^{\mathrm{T}}=\left[\hat{\mathbf{x}}_{k \mid k-1}^{\mathrm{T}}, \mathbf{0}_{n_{w} \times 1}^{\mathrm{T}}, \mathbf{0}_{n_{v} \times 1}^{\mathrm{T}}\right]^{\mathrm{T}}$ and covariance $\operatorname{matrix} \mathbf{P}_{k \mid k}^{A} x^{A} x=\operatorname{blkdiag}\left[\mathbf{P}_{k \mid k}^{x x}, \Sigma_{k}^{w}, \Sigma_{k+1}^{v}\right]$.

Note that the dimension of the augmented state is $n_{a}=n_{x}+$ $n_{w}+n_{v}$. The algorithm of the FAUKF will not be described here for brevity purposes. Its scheme is depicted in Fig. 2. Note that although the FAUKF requires only a single generation of the $\sigma$ points, at the end of each time step the $\sigma$-points are transformed to filtering moments.

\subsection{Fully Augmented UKF with reformulated correction step}

The FAUKF algorithm has been modified by Kolås et al. (2009) to improve numerical behavior and allow flexible constraint handling. The FAUKF with reformulated correction step is described by Algorithm 2 and illustrated in Fig. 3. For simplification purposes, the initialization step will be omitted henceforth.

Algorithm 2: Fully Augmented Unscented Kalman Filter with reformulated correction step

Step 1: (prediction) Calculate the $\sigma$-points

$$
\begin{aligned}
{ }^{A} \mathcal{X}_{k-1 \mid k-1}^{0: 2 n_{a}}= & \hat{\mathbf{x}}_{k-1 \mid k-1} \mathbf{1}_{1 \times b} \\
& +c\left[\mathbf{0}_{n_{a} \times 1}, \sqrt{\mathbf{P}_{k-1 \mid k-1}^{A} x^{A} x},-\sqrt{\mathbf{P}_{k-1 \mid k-1}^{A} x^{A} x}\right]
\end{aligned}
$$

with corresponding weights

$$
\left[{ }^{A} \mathcal{W}^{0}, \ldots,{ }^{A} \mathcal{W}^{2 n_{a}}\right]=\frac{1}{n_{a}+\kappa}\left[\kappa, \frac{1}{2}, \ldots, \frac{1}{2}\right],
$$

where $b=2 n_{a}+1$ and $c=\sqrt{n_{a}+\kappa}$. Propagate each $\sigma$-point through $\mathbf{f}_{k-1}$ as

$$
X_{k \mid k-1}^{i}=\mathbf{f}_{k-1}\left({ }^{x} X_{k-1 \mid k-1}^{i}\right)+{ }^{w} X_{k-1 \mid k-1}^{i}, \forall i
$$

and calculate the state predictive moments as

$$
\begin{aligned}
\hat{\mathbf{x}}_{k \mid k-1} & =\sigma-\operatorname{MEAN}\left({ }^{A} \mathcal{W}, X_{k \mid k-1}\right), \\
\mathbf{P}_{k \mid k-1}^{x x} & =\sigma-\operatorname{COV}\left({ }^{A} \mathcal{W}, X_{k \mid k-1}, X_{k \mid k-1}\right),
\end{aligned}
$$

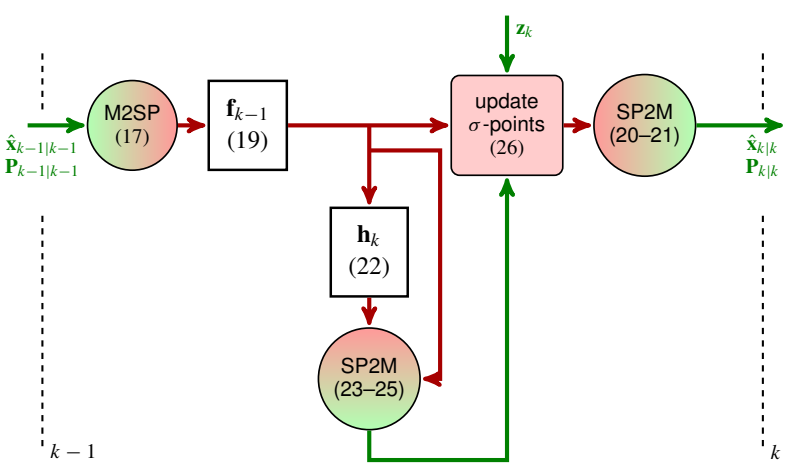

Fig. 3. Block scheme of the FAUKF algorithm with reformulated correction step.

Step 2: (filtering) Propagate each $\sigma$-point through $\mathbf{h}_{k}$ as

$$
Z_{k \mid k-1}^{i}=\mathbf{h}_{k}\left(\mathcal{X}_{k \mid k-1}^{i}\right)+{ }^{v} \mathcal{X}_{k-1 \mid k-1}^{i}, \forall i
$$

and calculate the measurement predictive moments as

$$
\begin{aligned}
& \hat{\mathbf{z}}_{k \mid k-1}=\sigma-\operatorname{MEAN}\left({ }^{A} \mathcal{W}, \mathfrak{Z}_{k \mid k-1}\right), \\
& \mathbf{P}_{k \mid k-1}^{z z}=\sigma-\operatorname{COV}\left({ }^{A} \mathcal{W}, \mathfrak{Z}_{k \mid k-1}, \mathfrak{Z}_{k \mid k-1}\right), \\
& \mathbf{P}_{k \mid k-1}^{x z}=\sigma-\operatorname{COV}\left({ }^{A} \mathcal{W}, \mathcal{X}_{k \mid k-1}, \mathfrak{Z}_{k \mid k-1}\right) .
\end{aligned}
$$

The filtering $\sigma$-points are computed according to

$$
\chi_{k \mid k}=X_{k \mid k-1}^{i}+\mathbf{K}_{k}\left(\mathbf{z}_{k}-Z_{k \mid k-1}^{i}\right),
$$

where $\mathbf{K}_{k}=\mathbf{P}_{k \mid k-1}^{x z}\left(\mathbf{P}_{k \mid k-1}^{z z}\right)^{-1}$. The filtering mean and covariance matrix are computed by

$$
\begin{aligned}
\hat{\mathbf{x}}_{k \mid k} & =\sigma-\operatorname{MEAN}\left({ }^{A} \mathcal{W}, \mathcal{X}_{k \mid k}\right), \\
\mathbf{P}_{k \mid k}^{x x} & =\sigma-\operatorname{COV}\left({ }^{A} \mathcal{W}, \mathcal{X}_{k \mid k}, \mathcal{X}_{k \mid k}\right),
\end{aligned}
$$

As can be seen, transformation of the updated $\sigma$-points to the moments is still inevitable to prevent an increase of the number of the $\sigma$-points (note that there are $n_{a} \sigma$-points after the filtering step). Hence the high order moments are again discarded.

Based on the above algorithms, the goal of the paper is to develop a UKF-based algorithm that allows propagation of the $\sigma$-points while keeping the higher order moment information. Keeping the higher order moments should lead to more precise first two moments and thus to increased accuracy.

As the algorithm will not augment the state (to keep the number of $\sigma$-points constant), it is necessary to design a technique that will modify the $\sigma$-point set to increase its covariance given by the contribution of the state and measurement noises. Such technique is a cornerstone of the proposed algorithm and will be adopted in moment update steps in (9) and (16) of Algorithm 1.

\section{SIGMA POINTS COVARIANCE INCREASE}

The technique to increase covariance of the $\sigma$-point set by their direct modification is based on the following theorem:

Theorem 1. Given a set of $\sigma$-points $X^{i} \in \mathbb{R}^{n_{x}}, i=0, \ldots 2 n_{x}$, their weights $\mathcal{W}^{0: 2 n_{x}}=\frac{1}{n_{x}+\kappa}\left[\kappa, \frac{1}{2}, \ldots, \frac{1}{2}\right]$ for which

$$
\begin{gathered}
\sigma-\operatorname{MEAN}(\mathcal{W}, \mathcal{X})=\hat{\mathbf{x}} \\
i \sigma-\operatorname{COV}(\mathcal{W}, \mathcal{X}, \mathcal{X})=\mathbf{P}^{x x}
\end{gathered}
$$

holds, let $\mathbf{U} \in \mathbb{R}^{n_{x} \times n_{x}}$ be a solution to the continuous Riccati equation

$$
2 \mathbf{U} \mathbf{U}^{\mathrm{T}}+\mathbf{U} \tilde{\mathbf{X}}^{\mathrm{T}}+\tilde{\mathbf{X}} \mathbf{U}^{\mathrm{T}}=2\left(n_{x}+\kappa\right) \mathbf{Q},
$$


where $\tilde{\mathbf{X}}=\mathcal{X}^{1: n_{x}}-\mathcal{X}^{n_{x}+1: 2 n_{x}}$. Then

$$
\begin{aligned}
& \sigma-\operatorname{MEAN}(\boldsymbol{w}, \mathcal{x}+\mathcal{u})=\hat{\mathbf{x}} \\
& \sigma-\operatorname{COV}(\boldsymbol{w}, \mathcal{x}+\mathcal{X}, \mathcal{x}+\mathcal{u})=\mathbf{P}^{x x}+\mathbf{Q},
\end{aligned}
$$

holds with

$$
u^{i}= \begin{cases}\mathbf{0}_{n_{x} \times 1} & i=0 \\ \mathbf{U} \mathbf{e}_{i} & i=1, \ldots, n_{x} \\ -\mathbf{U e}_{i-n_{x}} & i=n_{x}+1, \ldots, 2 n_{x},\end{cases}
$$

where $\mathbf{e}_{i}$ is the $i$-th column of $\mathbf{I}_{n_{x} \times n_{x}}$.

The proof can be found in Appendix A.

Based on the theorem, the $\sigma$-points can be modified to increase their covariance matrix by solving the Riccati equation and modifying each $\sigma$-point $\mathcal{X}^{i}$ by adding $U^{i}$. Further, this technique will be referred to as SPCI ( $\sigma$-point covariance increase).

Note that the increase of covariance of the $\sigma$-point set by their modification is not unique and the SPCI technique is just a simple and computationally efficient way.

\section{PURE PROPAGATION UKF}

Now, having the SPCI technique at disposal, it is possible to replace relations $(8-10)$ of the UKF by a direct modification of the $\sigma$-points. Such procedure will not discard higher order moments of the original $\sigma$-point set ${ }^{1}$. Hence, having the filtering $\sigma$-points $\mathcal{X}_{k \mid k}^{i}$, the predictive $\sigma$-points can be obtained by

$$
X_{k \mid k-1}^{i}=\mathbf{f}_{k}\left(X_{k-1 \mid k-1}^{i}\right)+U^{i}
$$

where the corrections $\mathcal{U}^{i}$ are obtained by the SPCI technique with $\mathbf{Q}=\Sigma_{k}^{w}$.

Further, following the idea of the FAUKF with reformulated correction step, in the filtering step it is possible to use (15) to modify the $\sigma$-points directly instead modifying the moments by (15) and (16). So when using the relation

$$
X_{k \mid k}^{i}=X_{k \mid k-1}^{i}+\mathbf{K}_{k}\left(\mathbf{z}_{k}-\mathcal{Z}_{k \mid k-1}^{i}\right),
$$

it holds that the mean of the $\sigma$-points is (Kolås et al., 2009)

$$
\sigma-\operatorname{MEAN}\left(\mathcal{W}_{k \mid k}, \mathcal{X}_{k \mid k}\right)=\hat{\mathbf{x}}_{k \mid k} \text {. }
$$

However, the covariance matrix of the $\sigma$-points equals to

$$
\sigma-\operatorname{COV}\left(\mathcal{W}_{k \mid k}, \mathcal{X}_{k \mid k}, \mathcal{X}_{k \mid k}\right)=\mathbf{P}_{k \mid k}^{x x}-\mathbf{K}_{k} \Sigma_{k}^{v} \mathbf{K}_{k} .
$$

To correct the filtering covariance matrix of the $\sigma$-points to be $\mathbf{P}_{k \mid k}^{x x}$, the SPCI technique must be used again with $\mathbf{Q}=$ $\mathbf{K}_{k}^{\mathrm{T}} \Sigma_{k}^{v} \mathbf{K}_{k}$.

Now, having all the prerequisites, it is possible to specify the algorithm of the UKF which preserves the $\sigma$-points representation of the state estimate for all time instants. As the algorithm is based on $\sigma$-point propagation only, it will be referred to as the pure propagation unscented Kalman filter (PPUKF).

\section{Algorithm 3: Pure Propagation Unscented Kalman Filter}

Step 1: (initialization) Set the time instant $k=0$.

Step 2: (prediction)

1 Note that the higher order moments might be affected by the way how the SPCI is implemented as the proposed SPCI is not a unique solution of the $\sigma$ points modification problem

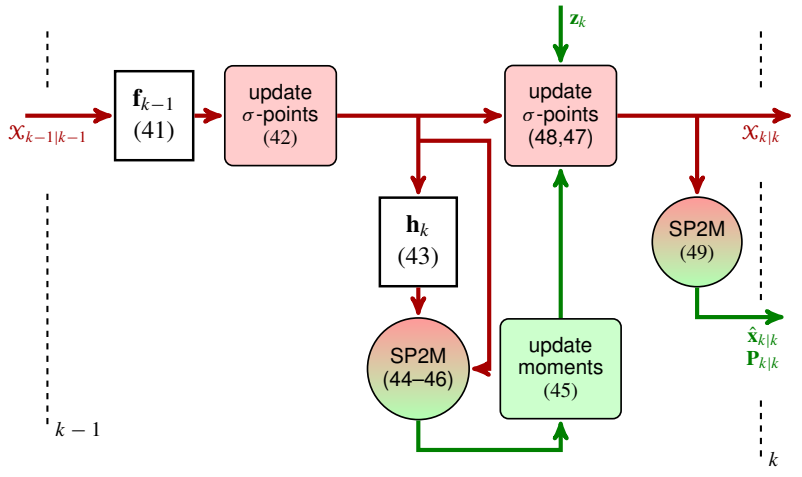

Fig. 4. Block scheme of the PPUKF algorithm.

If $k=0$, let $\hat{\mathbf{x}}_{0 \mid-1}=\mathrm{E}\left[\mathbf{x}_{0}\right]=\overline{\mathbf{x}}_{0}, \mathbf{P}_{0 \mid-1}^{x x}=\operatorname{cov}\left[\mathbf{x}_{0}\right]=\mathbf{P}_{0}^{x x}$ and calculate the $\sigma$-points

$$
x_{0 \mid-1}^{0: 2 n_{x}}=\hat{\mathbf{x}}_{0 \mid-1} \mathbf{1}_{1 \times b}+c\left[\mathbf{0}_{n_{x} \times 1}, \sqrt{\mathbf{P}_{0 \mid-1}^{x x}},-\sqrt{\mathbf{P}_{0 \mid-1}^{x x}}\right]
$$

with corresponding weights

$$
\mathcal{W}^{0: 2 n_{x}}=\frac{1}{n_{x}+\kappa}\left[\kappa, \frac{1}{2}, \ldots, \frac{1}{2}\right]
$$

where $b=2 n_{x}+1$ is the number of $\sigma$-points and $c=\sqrt{n_{x}+\kappa}$.

If $k>0$, propagate each $\sigma$-point through $\mathbf{f}_{k-1}$ as

$$
\bar{X}_{k \mid k-1}^{i}=\mathbf{f}_{k-1}\left(X_{k-1 \mid k-1}^{i}\right) \forall i
$$

and calculate the corrections $U_{k \mid k-1}^{i}$ of the $\sigma$-points by the SPCI technique with $X^{i}=\bar{X}_{k \mid k-1}^{i}$ and $\mathbf{Q}=\Sigma_{k}^{w}$. The predictive $\sigma$-points are given as

$$
x_{k \mid k-1}^{i}=\bar{X}_{k \mid k-1}^{i}+U_{k \mid k-1}^{i}=\mathbf{f}_{k}\left(X_{k-1 \mid k-1}^{i}\right)+u_{k \mid k-1}^{i}
$$

Step 3: (filtering) Propagate each $\sigma$-point through $\mathbf{h}_{k}$ as

$$
\mathcal{Z}_{k \mid k-1}^{i}=\mathbf{h}_{k}\left(\mathcal{X}_{k \mid k-1}^{i}\right), \forall i
$$

and calculate the measurement predictive moments as

$$
\begin{aligned}
\hat{\mathbf{z}}_{k \mid k-1} & =\sigma-\operatorname{MEAN}\left(\mathcal{W}, \mathfrak{Z}_{k \mid k-1}\right), \\
\mathbf{P}_{k \mid k-1}^{z z} & =\sigma-\operatorname{COV}\left(\mathcal{W}, \mathfrak{Z}_{k \mid k-1}, \mathfrak{Z}_{k \mid k-1}\right)+\Sigma_{k}^{v}, \\
\mathbf{P}_{k \mid k-1}^{x z} & =\sigma-\operatorname{COV}\left(\mathcal{W}, \boldsymbol{X}_{k \mid k-1}, \mathfrak{Z}_{k \mid k-1}\right) .
\end{aligned}
$$

Propagate the predictive $\sigma$-points through the relation

$$
\bar{X}_{k \mid k}^{i}=X_{k \mid k-1}^{i}+\mathbf{K}_{k}\left(\mathbf{z}_{k}-\mathcal{Z}_{k \mid k-1}^{i}\right)
$$

and calculate the corrections $U_{k \mid k}^{i}$ of the $\sigma$-points by the SPCI technique with $\mathcal{X}^{i}=\bar{\chi}_{k \mid k}^{i}$ and $\mathbf{Q}=\mathbf{K}_{k}^{\mathrm{T}} \Sigma_{k}^{v} \mathbf{K}_{k}$. The filtering $\sigma$-points are given as

$$
\chi_{k \mid k}^{i}=\bar{\chi}_{k \mid k}^{i}+u_{k \mid k}^{i}
$$

The state estimate $\hat{\mathbf{x}}_{k \mid k}$ can computed as

$$
\hat{\mathbf{x}}_{k \mid k}=\sigma-\operatorname{MEAN}\left(\mathcal{W}, X_{k \mid k}\right)
$$

Note that if requested, any higher order moment can be calculated using the $\sigma$-points $\mathcal{X}_{k \mid k}^{i}$ and weights $\boldsymbol{W}^{i}$.

Let $k=k+1$. The algorithm then continues by Step 2 .

As can be seen, the PPUKF propagates the $\sigma$-points of the state, without any re-computation of the $\sigma$-points based on moments. Block scheme of the PPUKF algorithm is depicted in Fig. 4.

\section{NUMERICAL ILLUSTRATION}

The newly proposed PPUKF will be illustrated in an example dealing with bearings-only tracking (Ristic et al., 2004). The 
target follows a course of $-140^{\circ}$ starting $12 \mathrm{~km}$ away from the platform at a constant speed of 4 knots. The platform follows a course of $140^{\circ}$ at a constant speed of 5 knots and at $k=13$ executes a maneuver to reach a new course of $18^{\circ}$. The initial positions are $[12 \mathrm{~km}, 2 \mathrm{~km}]$ for the target and $[0,0]$ for the platform. The model was simulated for 40 minutes and the geometry of the motion is depicted in Fig. 5. For the estimation

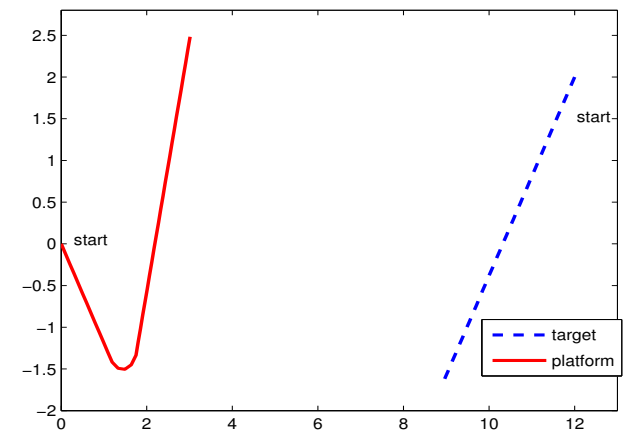

Fig. 5. Simulation scenario.

purposes, the object state is given by $\mathbf{x}_{k} \triangleq\left[x_{k}, y_{k}, \dot{x}_{k}, \dot{y}_{k}\right]^{\mathrm{T}}$ (i.e. it consists of the positions and velocities in the $x$ and $y$ directions and evolves according to the continuous white noise acceleration motion model

$$
\mathbf{x}_{k+1}=\left[\begin{array}{llll}
1 & 0 & T & 0 \\
0 & 1 & 0 & T \\
0 & 0 & 1 & 0 \\
0 & 0 & 0 & 1
\end{array}\right] \mathbf{x}_{k}-\mathbf{V}_{k, k+1}+\left[\begin{array}{cc}
0.5 T^{2} & 0 \\
0 & 0.5 T^{2} \\
T & 0 \\
0 & T
\end{array}\right] \mathbf{w}_{k},
$$

where $T=1 \mathrm{~min}$ is the sampling interval, $\mathbf{V}_{k, k+1}$ is a vector of deterministic inputs accounting for the effect of the platform acceleration (Ristic et al., 2004), and the covariance matrix of the state noise is $\Sigma^{w}=1.6 \times 10^{-6} \mathbf{I}_{2 \times 2} \mathrm{~km}^{2} / \mathrm{s}^{4}$.

The object is observed from a platform, which provides the measurement $z_{k}$ at time $k$ given by the angle from the platform to the object. Suppose that at time $k$ the platform is located at coordinates $\left[x_{k}^{p}, y_{k}^{p}\right]$, then the measurement $z_{k}$ is given by

$$
z_{k}=\arctan \frac{x_{k}-x_{k}^{p}}{y_{k}-y_{k}^{p}}+v_{k},
$$

where the variance of the measurement noise is $\Sigma^{v}=\left(1.5^{\circ}\right)^{2}$.

Four filters were tested: UKF, FAUKF, FAUKF with reformulated correction step (FAUKF-RCS), and PPUKF using $N=10^{3}$ Monte Carlo (MC) runs. They were initialized according to Ristic et al. (2004) with initial range $r, p(r)=$ $\mathcal{N}\left\{r ; \sqrt{12^{2}+2^{2}}, 4^{2}\right\}$ and speed $s, p(s)=\mathcal{N}\left\{s ; \bar{s},(2 \cdot 0.03)^{2}\right\}$, where $\bar{s}$ is true speed.

Their performance was compared using the root mean square error (RMSE) defined as

$$
\operatorname{RMSE}_{k}^{\mathrm{x}}=\sqrt{\frac{1}{N} \sum_{i=1}^{N}\left(\hat{x}_{k}^{i}-x_{k}^{i}\right)^{2}}
$$

for the $x$ position error, where $x_{k}^{i}$ and $\hat{x}_{k}^{i}$ denote true and estimated target positions at the $i$-th MC run. The RMSE for the $y$ direction is calculated analogically to (50). The RMSEs for both coordinates are depicted in Fig 6. Performance of the filters was also compared using the average normalized estimation error squared (ANEES) measure defined as ( $\mathrm{Li}$ and Zhao, 2006)

$$
\operatorname{ANEES}_{k}=\frac{1}{n_{x} N} \sum_{i=1}^{N}\left(\left(\mathbf{x}_{k}^{i}-\hat{\mathbf{x}}_{k}^{i}\right)^{\mathrm{T}}\left(\mathbf{P}_{k \mid k}^{i}\right)^{-1}\left(\mathbf{x}_{k}^{i}-\hat{\mathbf{x}}_{k}^{i}\right)\right),
$$

where $\mathbf{P}_{k \mid k}^{i}$ denotes the filtering covariance matrix provided by the filters in $i$-the MC simulation. The ANEES values are depicted in Fig 7. The results indicate that the PPUKF achieves
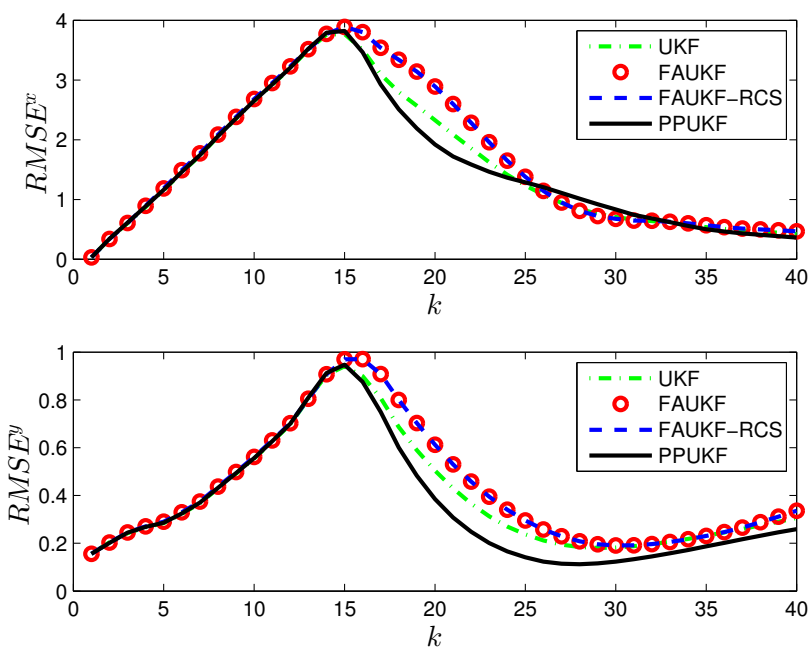

Fig. 6. RMSE for position estimates versus time for the UKF, FAUKF, FAUKF-RCS and PPUKF.

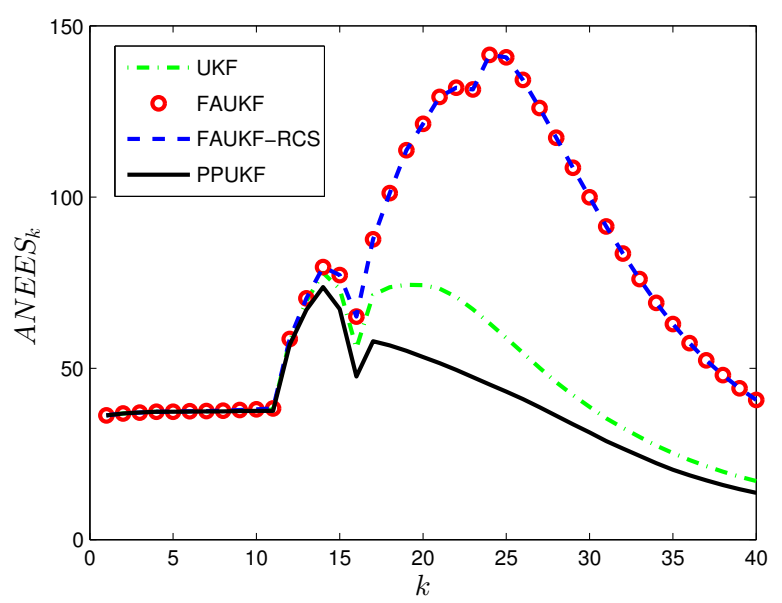

Fig. 7. ANEES versus time for the UKF, FAUKF, FAUKF-RCS and PPUKF.

the lowest RMSE which is caused by propagation of the $\sigma$ points without their re-computation based on moments. This leads to preservation of higher order moments and consequently to an increased precision of the mean. This is confirmed by the ANEES which evaluates not only the error of the state estimate but also the covariance matrix produced by the filter.

Note that the RMSE increase in $k \in(0,13)$ is given by a low observability of the state. A change of the object position in this period is reflected in the measurement negligibly.

To illustrate preservation of the higher-order moments, estimates of the third central moment of the state were computed 
Table 1. Computational costs of a single step of the filters.

\begin{tabular}{c||cccc} 
& UKF & FAUKF & FAUKF-RCS & PPUKF \\
\hline \hline Time $[\mathrm{msec}]$ & 0.26 & 0.30 & 0.33 & 2.00
\end{tabular}

(further denoted as $M_{k \mid k}^{\mathbf{x x x}, i}$ and representing a column of third moments of individual state variables at the $i$ th $\mathrm{MC}$ run) and an average of their absolute values sum over the MC runs given by

$$
M_{3, k}=\frac{1}{N} \sum_{i=1}^{N} \mathbf{1}_{1 \times n_{x}}\left|M_{k \mid k}^{\mathbf{x x x}, i}\right|,
$$

is depicted in Fig 8. From the figure it follows that the PPUKF

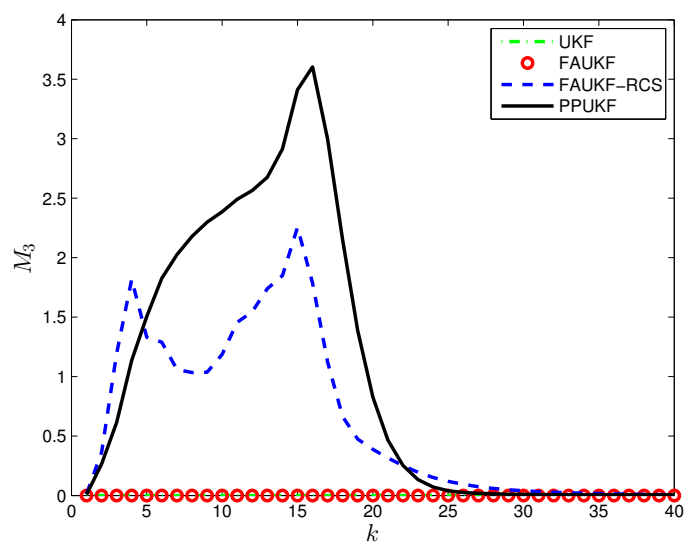

Fig. 8. Average of sum of third moments of state variables versus time for the UKF, FAUKF, FAUKF-RCS and PPUKF.

is capable of capturing third order moment of the state better than the FAUKF-RCS which recomputes the $\sigma$-points at each time instant. The UKF and FAUKF provide only recomputed filtering $\sigma$-points, hence their third moment is zero.

Computational costs of a time step are given in Table 1. The increased costs of the PPUKF result from the Riccati equation solved in the SPCI technique.

\section{CONCLUDING REMARKS}

The paper dealt with state estimation of nonlinear stochastic systems with the focus on the UKF. A new UKF-based filter with pure propagation of the $\sigma$-points has been proposed. The proposed algorithm completely eliminates re-computation of the $\sigma$-points executed in classical UKF algorithms for the purpose of correction of the moments. To this end a new technique for a direct modification of the $\sigma$-points has been developed. The results of a bearings-only tracking example indicate increased estimate quality of the proposed PPUKF at the cost of higher computational costs caused mainly by the proposed technique for direct modification of the $\sigma$-points.

\section{REFERENCES}

I. Arasaratnam and S. Haykin. Cubature Kalman filters. IEEE Transactions on Automatic Control, 54(6):1254-1269, 2009.

S. Arulampalam, S. Maskell, N. Gordon, and T. Clapp. A tutorial on particle filters for on-line non-linear/non-Gaussian Bayesian tracking. IEEE Transactions on Signal Processing, (2):174-188, 2002.

J. Duník, M. Šimandl, and O. Straka. Unscented Kalman filter: Aspects and adaptive setting of scaling parameter. IEEE Transactions on Automatic Control, 57(9):2411-2416, 2012.
K. Ito and K. Xiong. Gaussian filters for nonlinear filtering problems. IEEE Transactions on Automatic Control, 45(5): 910-927, 2000.

B. Jia, M. Xin, and Y. Cheng. Sparse Gauss-Hermite quadrature filter with application to spacecraft attitude estimation. Journal of Guidance, Control, and Dynamics, 34(2), 2011.

S. J. Julier, J. K. Uhlmann, and H. F. Durrant-Whyte. A new method for the nonlinear transformation of means and covariances in filters and estimators. IEEE Transactions on Automatic Control, 45(3):477-482, 2000.

R. E. Kalman. New results in linear filtering and prediction theory. Journal of Basic Engineering, pages 34-45, 1960. Transactions ASME.

S. Kolås, B. A. Foss, and T. S. Schei. Constrained nonlinear state estimation based on the UKF approach. Computers and Chemical Engineering, 33(8):1386-1401, 2009.

X. R. Li and Z. Zhao. Measuring estimators credibility: Noncredibility index. In Proceedings of 2006 International Conference on Information Fusion, Florence, Italy, 2006.

M. Norgaard, N. K. Poulsen, and O. Ravn. New developments in state estimation for nonlinear systems. Automatica, 36 (11):1627-1638, 2000.

B. Ristic, S. Arulampalam, and N. Gordon. Beyond the Kalman Filter: Particle Filters for Tracking Applications. Artech House, 2004.

J. Sarmavuori and S. Sarkka. Fourier-Hermite Kalman filter. IEEE Trans. on Automatic Control, 57(6):1511-1515, 2012.

M. Šimandl and J. Duník. Derivative-free estimation methods: New results and performance analysis. Automatica, 45(7): 1749-1757, 2009.

R. van der Merwe. Sigma-Point Kalman Filters for Probabilistic Inference in Dynamic State-Space Models. PhD thesis, OGI School of Science \& Engineering, Oregon Health \& Science University, Portland, OR, USA, April 2004.

\section{Appendix A. PROOF OF LEMMA 1}

Proof of the first part (32):

$$
\begin{aligned}
& \sum_{i=0}^{2 n_{x}} \mathcal{W}^{i}\left(\mathcal{X}^{i}+\mathcal{U}^{i}\right)=\sum_{i=0}^{2 n_{x}} \mathcal{W}^{i} \mathcal{X}^{i}+\sum_{i=0}^{2 n_{x}} \mathcal{W}^{i} u^{i} \\
& =\hat{\mathbf{x}}+\sum_{i=0}^{2 n_{x}} \mathcal{W}^{i} \mathcal{U}^{i}=\hat{\mathbf{x}}+\mathcal{W}^{0} \mathbf{0}_{n_{x} \times 1}+\sum_{i=1}^{2 n_{x}} \mathcal{W}^{i} u^{i} \\
& =\hat{\mathbf{x}}+\sum_{i=1}^{n_{x}} \mathcal{W}^{i} \mathbf{U} \mathbf{e}_{i}+\sum_{i=n_{x}+1}^{2 n_{x}} \mathcal{W}^{i}\left(-\mathbf{U} \mathbf{e}_{i-n_{x}}\right)=\hat{\mathbf{x}}
\end{aligned}
$$

Proof of the second part (33): For convenient purposes define $\bar{X}^{i} \triangleq X^{i}-\hat{\mathbf{x}}$ and $\alpha=2\left(n_{x}+\kappa\right)$. Then

$$
\begin{aligned}
& \sum_{i=0}^{2 n_{x}} W^{i}\left(\bar{X}^{i}+U^{i}\right)\left(\bar{X}^{i}+U^{i}\right)^{\mathrm{T}}=\sum_{i=0}^{2 n_{x}} w^{i} \bar{X}^{i}\left(\bar{X}^{i}\right)^{\mathrm{T}} \\
& +\sum_{i=0}^{2 n_{x}} w^{i}\left(\bar{X}^{i}\left(\mathcal{U}^{i}\right)^{\mathrm{T}}+\mathcal{U}^{i}\left(\bar{X}^{i}\right)^{\mathrm{T}}+\mathcal{U}^{i}\left(u^{i}\right)^{\mathrm{T}}\right) \\
& =\mathbf{P}^{x x}+\mathcal{W}^{0}\left(\bar{X}^{0}\left(\mathcal{U}^{0}\right)^{\mathrm{T}}+\mathcal{U}^{0}\left(\bar{\chi}^{0}\right)^{\mathrm{T}}+\mathcal{U}^{0}\left(u^{0}\right)^{\mathrm{T}}\right) \\
& +\frac{1}{\alpha} \sum_{i=1}^{2 n_{x}}\left(\overline{\mathcal{X}}^{i}\left(u^{i}\right)^{\mathrm{T}}+u^{i}\left(\overline{\mathcal{X}}^{i}\right)^{\mathrm{T}}+U^{i}\left(\mathcal{U}^{i}\right)^{\mathrm{T}}\right) \\
& =\mathbf{P}^{x x}+\frac{1}{\alpha} \sum_{i=1}^{2 n_{x}}\left(\bar{X}^{i}\left(\mathbf{U e}_{i}\right)^{\mathrm{T}}+\mathbf{U} \mathbf{e}_{i}\left(\overline{\mathcal{X}}^{i}\right)^{\mathrm{T}}+\mathbf{U} \mathbf{e}_{i}\left(\mathbf{U} \mathbf{e}_{i}\right)^{\mathrm{T}}\right) \\
& =\mathbf{P}^{x x}+\frac{1}{\alpha} \sum_{i=1}^{n_{x}}\left(\left(\bar{X}^{i}-\bar{X}^{i+n x}\right)\left(\mathbf{U e}_{i}\right)^{\mathrm{T}}+\mathbf{U e}_{i}\left(\bar{X}^{i}-\bar{X}^{i+n_{x}}\right)^{\mathrm{T}}\right. \\
& \left.+2 \mathbf{U} \mathbf{e}_{i}\left(\mathbf{U} \mathbf{e}_{i}\right)^{\mathrm{T}}\right)=\mathbf{P}^{x x}+\frac{1}{\alpha}\left(\left(\mathcal{X}^{1: n_{x}}-\mathcal{X}^{n_{x}+1: 2 n_{x}}\right)(\mathbf{U})^{\mathrm{T}}\right. \\
& \left.+\mathbf{U}\left(\mathcal{X}^{1: n_{x}}-\mathcal{X}^{n_{x}+1: 2 n_{x}}\right)^{\mathrm{T}}+2 \mathbf{U}^{\mathrm{T}}\right)= \\
& \mathbf{P}^{x x}+\frac{1}{\alpha}\left(\mathbf{U} \tilde{\mathbf{X}}^{\mathrm{T}}+\tilde{\mathbf{X}} \mathbf{U}^{\mathrm{T}}+2 \mathbf{U} \mathbf{U}^{\mathrm{T}}\right)=\mathbf{P}^{x x}+\frac{1}{\alpha} \alpha \mathbf{Q}=\mathbf{P}^{x x}+\mathbf{Q}
\end{aligned}
$$

